\title{
HIGHLIGHTS
}

PROSTATE CANCER

\section{The promise of psychostimulants for disease-related fatigue}

The first randomized placebo-controlled trial of a psychostimulant for prostate cancer-related fatigue has generated encouraging preliminary results. Despite the difficulty they experienced recruiting participants, the study team remains upbeat about the potential of drugs of this type to improve patients' quality of life.

\section{1 ...the observed efficacy of psychostimulation provides a sound basis for pursuing larger trials 77}

Up to $70 \%$ of men with prostate cancer are distressed by excessive tiredness. Andrew Roth and colleagues therefore assumed that enrolling 120 ambulatory patients from the 1,000-plus being treated at Memorial Sloan-Kettering Cancer Center in New York was a realistic goal.

Unfortunately, only 32 men from a pool of almost 250 with moderate or severe fatigue made themselves available for randomization. Many "were fearful about potential side effects".

Are such concerns warranted? Six of the 16 men who were eventually randomized to receive dose-escalated methylphenidate dropped out of the study. Tachycardia and increased blood pressure were the adverse effects responsible for these withdrawals.

Participants who completed the 6-week trial reported markedly improved energy levels. Most of those who tolerated methylphenidate expressed a desire to continue taking the "wonder drug".

Interestingly, men in the placebo group also experienced a clinically significant amelioration of fatigue. The magnitude of the placebo effect tended, however, to be less than that of methylphenidate.

The investigators assert that the observed efficacy of psychostimulation provides a sound basis for pursuing larger trials. They speculate that testing a drug with a superior cardiovascular adverse-effect profile or a longer-acting agent might enhance retention of trial volunteers.
Long-acting methylphenidate was trialed recently in a population of patients with various types of cancer. The 148strong study cohort included a small number of men with prostate cancer.

After 4 weeks, fatigue scores had improved in both the active and control arms. However, there was no significant difference in response between the two groups. Adverse effects were reported more frequently by patients taking methylphenidate.

Careful consideration of risks versus benefits will be necessary before effort is expended on new trials of psychostimulants in prostate cancer.

Suzanne J. Farley

Original articles Roth, A. J. et al. Methylphenidate for
fatigue in ambulatory men with prostate cancer. Cancer
doi:10.1002/cncr.25424 | Moraska, A. R. et al. Phase III,
randomized, double-blind, placebo-controlled trial of long-
acting methylphenidate for cancer-related fatigue: North
Central Cancer Treatment Group NCCTG-NO5C7 trial. J. Clin.
Oncol. doi:10.1200/JC0.2010.28.1444

Original articles Roth, A. J. et al. Methylphenidate for randomized, double-blind, placebo-controlled trial of longacting methylphenidate for cancer-related fatigue: North Oncol. doi:10.1200/JC0.2010.28.1444 